\title{
From the Loom to the Laboratory: Molecular Textiles
}

\author{
Alfredo Di Silvestro ${ }^{a}$ and Marcel Mayor ${ }^{\star a b c}$
}

\begin{abstract}
Weaving is an old technique producing fabric materials by interlocking yarns, which we appreciate every day by wearing textiles. The splendid mechanical features of these macroscopic interwoven structures such as stability, flexibility, and shape adaptability raised the question whether or not such properties might also be observed on a molecular level. In this article, molecular analogues to textiles are discussed and strategies to molecular weaves are presented. While there are impressive structural similarities between the macroscopic and the molecular world, molecular textiles consisting of interwoven linear polymers remain a challenge. The scope of the article ranges from discrete superstructures like Solomon knots, over deoxyribonucleic acid (DNA) based nanoscale patterns and interwoven $2 \mathrm{D}$ sheets of coordination polymers, to weaving strategies interlinking small organic precursors.
\end{abstract}

Keywords: 2D polymer $\cdot$ Interwoven network $\cdot$ Molecular textile $\cdot$ Molecular fabrics $\cdot$ Molecular weave

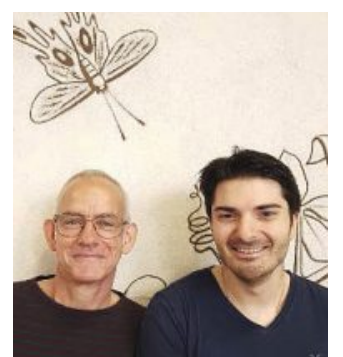

Alfredo Di Silvestro (right) was born in Basel (Switzerland) in 1991. He studied chemistry at the University of Basel and received his Master of Science in 2017 developing phthalocyanines with photocleavable tags. For his $\mathrm{PhD}$ he joined the group of Prof. Mayor at the University of Basel to study new concepts for molecular fabrics. Marcel Mayor (left) received his $\mathrm{PhD}$ in 1995 from the University of Bern under the supervision of Rolf Scheffold and Lorenz Walder. After working with Jean-Marie Lehn at the University Louis Pasteur in Strasbourg (France) and at the Collège de France in Paris (France), he founded his own research group in the Institute of Nanotechnology (INT) at the Karlsruhe Institute of Technology (KIT, Germany) in 1998. In 2004 he became Professor of Chemistry at the Department of Chemistry of the University of Basel (Switzerland), and in 2011 he became adjunct Professor of Chemistry of School of Chemistry of the Sun Yat-Sen University in Guangzhou (China). His current research interests are supramolecular chemistry, molecular electronics, nanoscale architectures, functional and hybrid materials.

\section{Introduction}

In our everyday lives, we are surrounded by two-dimensional materials with leaves, papers, carpets, and foils or even windows and tables as prominent examples. Particularly fascinating are fabric materials providing the flexibility required to adapt to curved shapes and thus play a key role in our society, e.g. as clothes keeping us warm by covering our bodies. ${ }^{[1,2]}$ The flexibility arises from their structure consisting of interwoven yarns. While the yarns can be very strong with impressive tensile strength, the fabric material remains flexible due to the mechanical nature of the interyarn interaction. ${ }^{[2,3]}$ The oldest and simplest fabrics are produced by two orthogonally interlaced yarns, ${ }^{[4]}$ an arrangement obtained by so called biaxial weaving (Fig. 1a). The vertical or lengthwise thread is called warp yarn and the horizontal is known as fill yarn or weft, pointing at their role in the production of the fabrics. ${ }^{[5]}$ In a loom the warp yarns are mounted parallel and the mechanics of the device allows altering the relative heights of the even and uneven warp yarns. The weft is 'filled' between the even and uneven warp yarns and after each application of the weft yarn, the relative heights of the even and uneven warp yarns are inverted. Thereby the biaxial woven fabric is obtained and it is the spatial control over the yarns provided by the loom that allows its assembly.

The mechanical features of textiles combining stability, flexibility and shape adaptability makes them not only a very successful family of materials, it also provokes the question whether the design concept might not also be applied on a molecular level. This article provides an overview how the concepts of fabric materials inspired chemists in their search for new materials a)

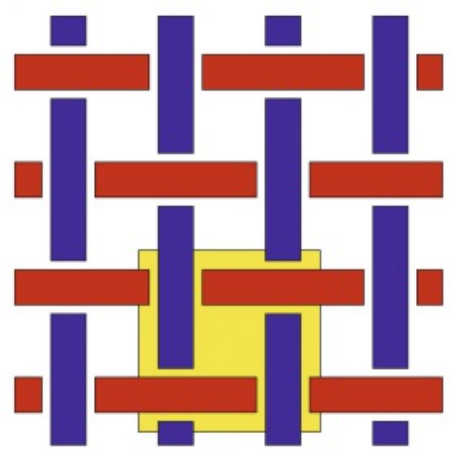

b)

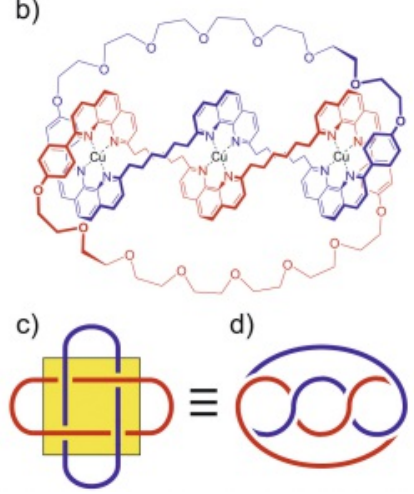

Fig. 1. a) Sketch of a biaxial woven fabric. b) Trinuclear $\mathrm{Cu}(\mathrm{II})$ complex resulting in a doubly interlocked [2]-catenane sketched in d) upon removal of the copper ions. c) Alternative representation of the doubly interlocked [2]-catenane as Solomon knot. The analogy of the Solomon knot with the unit cell of the fabric is highlighted by a yellow square. 
with unprecedented properties and novel behaviours. It is rather a collection of particular appealing examples representing the underlying concepts in a plausible manner than a comprehensive collection of reported achievements.

\section{Solomon Knots: Mechanically Interlinked Model Compounds}

With the rise of supramolecular chemistry in the past few decades, mechanically interlinked molecules gained increasing attention. And indeed, Solomon knots have been assembled consisting of two doubly interlinked macrocycles, which can be considered as the basic 'unit cell' of a fabric architecture (yellow square in Fig. 1a, and 1c). These mechanically interlinked superstructures are usually obtained by performing the macrocyclization(s) with suitably prearranged doubly wrapped precursors. In most cases, the required prearrangements of the reactants are based on supramolecular concepts like e.g. the engagement of the precursor in coordination compounds or the reliable design of multiple parallel hydrogen bonds in deoxyribonucleic acid (DNA) based model compounds. To the best of our knowledge, the first example of a Solomon knot was reported by Sauvage and coworkers profiting from the template effect of copper ions. ${ }^{[6]}$ A macrocycle comprising three phenanotroline binding sites formed a trinuclear doubly intertwined helical complex with the open chain precursor. Upon formation of the second macrocycle (displayed in Fig. 1b) and removal of the copper ions, the two macrocycles were mechanically interlocked forming a doubly interlocked [2]-catenane as superstructure (Fig. 1d), which corresponds to a Solomon knot (Fig. 1c and 1d).

In a more recent example, Solomon knots were obtained as only one of several examples of mechanically interlinked macrocycles forming knots. ${ }^{[7]}$ The reversible nature of the macrocyclization provided a dynamic combinatorial library of molecular knots in the solid state from which a particular species was favoured by the choice of coordinating ions and solvents. The Solomon knot of interest here crystallized only upon providing equal amounts of copper and zinc acetate. Solomon knots were also obtained from single-stranded DNA. ${ }^{[8]}$ Careful design of both the DNA sequence and helical chirality of two DNA strands enabled the formation of a pair of twofold catenated precursors forming macrocycles upon ligation. Interestingly the motivation of these Solomon knots was exclusively to explore the suitability of the technique to provide fabric-type structures pointing at the promising prospects related with mechanically interwoven molecular structures. However, these Solomon knots are only models of the mechanical junction between the yarns of a fabric and thus can be considered as proof of concept for the mechanical joint. They are reduced in dimension representing exclusively a pair of crossing yarns and lack the lateral repetition of the motive making fabrics the promising $2 \mathrm{D}$ materials.

\section{Fabric-type Interwoven Molecular Arrangements (in the crystalline state)}

\subsection{Deoxyribonucleic acid (DNA)-based Fabrics}

As already displayed above with the controlled formation of a Solomon knot, DNA is an ideal material for the programmed assembly of complex nanoscale $2 \mathrm{D}$ patterns, structures and even 3D objects. ${ }^{[9,10]}$ Customized DNA strands are accessible by automated synthesizers or by biotechnologies profiting from viral DNA syntheses. The predictable assembly due to Watson-Crick type base pairing combined with the geometrical stability and high persistence length of the obtained double helices enables the encoding of well-defined structures by molecular design. ${ }^{\left[{ }^{8]}\right.}$ Large numbers of identical spatially well-defined and shape-persistent nanoscale building blocks are self-assembled in solution and their periphery is decorated with single-stranded overhangs, socalled 'sticky-ends'. These 'sticky-ends' allow control over the subsequent assembly of the building blocks in periodic patterns, as the arrangement of the building blocks in the resulting solidstate crystal is dictated by both, the position of the 'sticky-end' at the surface of the building block and the encoded DNA sequence complementarity. The helicity of the double helix results in twisted DNA strands and the interlinking of the prearranged single-strands by formation of phosphodiester bonds results in mechanically interlinked macrocycles. Thereby $3 \mathrm{D}$ objects, as demonstrated by the pioneering proof of concept DNA cube, ${ }^{[11]}$ but also 2D periodic patterns can reliably be designed and subsequently assembled. Such laterally expanding 2D crystals are particular appealing as a proof of concept as their formation and dimensions are accessible by scanning probe techniques. The coating of a substrate with a periodic pattern of geometrically well-defined building blocks resembles the tiling of a floor and indeed, the pioneering 2D DNA striped lattice reported by Winfree and Seeman was inspired by the mathematical theory of Wang tiles, where tiles with similar coloured edges are placed next to each other. ${ }^{[12]}$ In Fig. 2 the geometrically simplest 2D interwoven framework is displayed, which requires only two different DNA tiles and the inspiring Wang tile theory can still be recognized in their representation of the approach (Fig. 2a). It is labelled DAO as abbreviation for double crossover, antiparallel, odd spacing describing the nature of the connections defined by the DNA tiles. In Figs $2 b$ and $2 \mathrm{c}$ each component oligonucleotide is displayed in a unique colour. While the crossover points are circled in Fig. 2b, the tiles defining the repeat units are highlighted by pale grey rectangle in Fig. 2c. The tile-type periodicity of the 2D object obtained by the approach can clearly be seen in the pictures obtained by atomic force microscopy (AFM) (Figs 2d and 2e). While these periodic lateral structures resemble in their shape a nanoscale fabric, the tiles are interlinked by base pairing of the sticky ends and thus, reversible supramolecular forces are holding the fabric together.
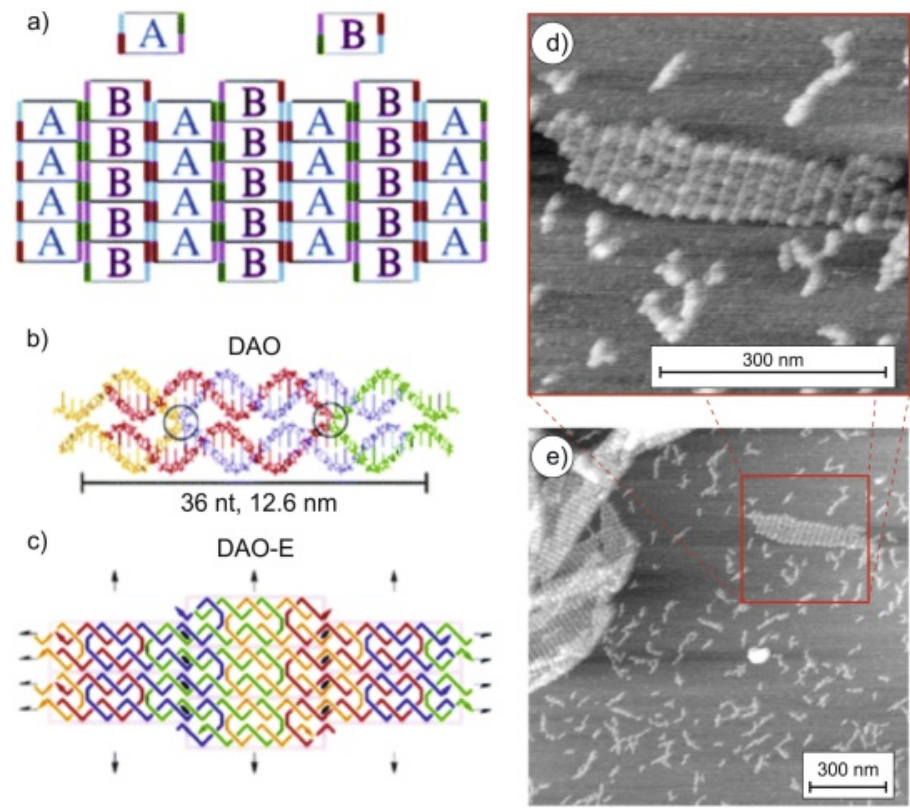

Fig. 2. 2D interwoven framework consisting of tailor-made DNA tiles inspired by the Wang tile theory. a) Sketch of the concept of tiling the surface with two different tiles exposing complementary attractive edges as indicated by the matching colours. The horizontal offset of tile B with respect to tile $A$ results in the $2 \mathrm{D}$ network. b) Illustration of DAO model structure for type A unit consisting of 36 nucleotides. Each component oligonucleotide is displayed in a unique colour. The crossover points are highlighted by circles. c) Sketch of the woven lattice produced by the DAO units. Each repeating unit is indicated by a pale grey rectangle. $\mathrm{d}, \mathrm{e})$ The same AFM image of a DAO-E AB fabric in two different magnifications clearly showing the formation of the 2D lattice. Adapted from ref. [12] with permission from Springer Nature. 
Two-dimensional DNA lattices became a mature technology enabling the spatial control of components on the nanoscale. Numerous fascinating aspects have been reported from various research groups and it would go beyond the scope of this article to provide a comprehensive overview. Also in analogy with most technologies, with increasing maturity the focus moves more and more towards their application potential away from fundamental structural aspects pointing at the interwoven fabric nature of their networks. This resembles very much our own appreciation of fabrics in the macroscopic world, where we are usually more interested in their properties (colours, cut, thickness, isolation features, etc.) than in the arrangement of the yarns resulting in the fabric. In the following only a very few examples of aspects related to fabrics will be discussed.

A particularly appealing example demonstrating the extent of lateral control over 2D DNA-based objects is the arrangement of long biological DNA strands by synthetic staple strands in well-defined shapes, ${ }^{[13]}$ called Origami design. The concept is sketched in Fig. 3a with the biologically obtained single scaffold strand (coloured grey and black) laterally fixed by the coloured short oligonucleotides called staple strands. The potential and versatility of the approach is demonstrated with the AFM figures of the smiley faces (Fig. 3d and 3e) obtained by programming the lateral fixation of the scaffold strand by suitable staple strands (Fig. 3b and 3c).

With the lateral periodicity as the main feature, DNA networks tend to behave as a fishing net lacking the lateral flexibility of a fabric. Their design is also not restricted to the biaxial weaving with perpendicular yarns and alternative networks have been reported. A recent example even demonstrates the sensitivity of the lattice formation on the presence of additional species. Adding a recombinatorial bacterial protein as intrinsic component of the network varies the geometrical features of the networks formed by the same four nucleotides. ${ }^{[14]}$ The bare four nucleotides form a so-called 'Holliday junction' exposing two complementary sets of sticky ends in a $\chi$-stacked junction with an angle of about $120^{\circ}$ (Fig. 4a) resulting in a Kagome lattice (Fig. 4b) with three interwoven DNA strands. Upon addition of the bacterial recombination protein (RuvA) the four nucleotides are arranged in a rectangular junction (Fig. 4d) resulting in a square planar lattice (Fig. 4e). The crystallized lattices have the dimensions and lateral periodicity to enable their observation by transmission electron microscopy (TEM) as displayed in Figs 4c and 4f. a)

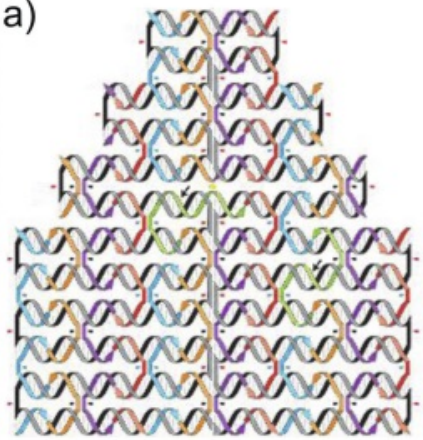

b)

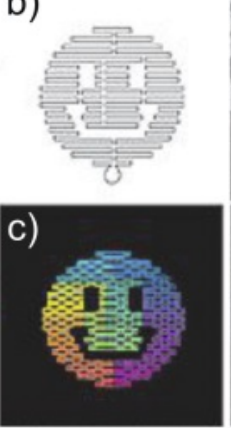

a)
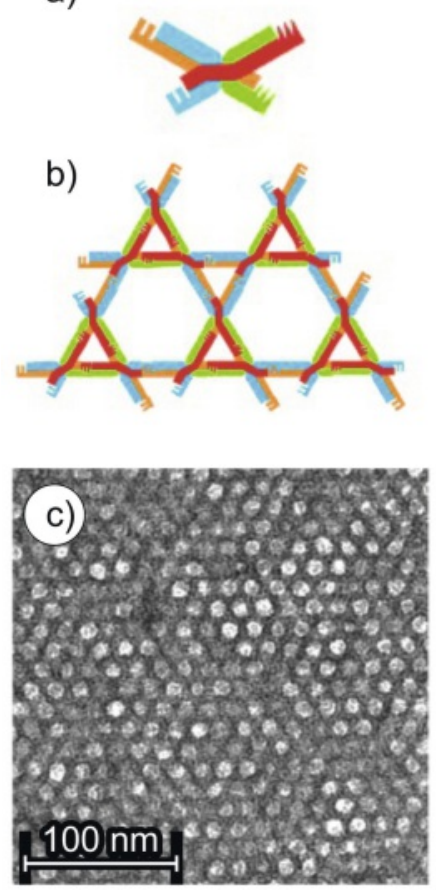

d)

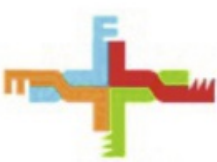

e)

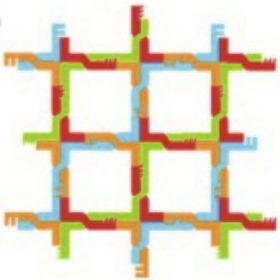

Fig. 4. 2D DNA framework based on 'Holliday junctions'. a) Schematic representation of a $\chi$-stacked junction composed of four nucleotides (red, blue, orange and green) with an angle of about $120^{\circ}$. The teeth at the end symbolize the complementary sticky ends. b) Sketch of the Kagome lattice formed by the assembly of $\chi$-stacked junctions and c) the corresponding TEM micrograph. d) Representation of the square planar junction formed in the presence of the bacterial protein (RuvA). e) Sketch of the square lattice and f) the corresponding TEM micrograph. Adapted from ref. [14] with permission from John Wiley and Sons.

An increasing number of beautiful examples display the potential of net-type supramolecular DNA fabrics in controlling the spatial arrangement at the nanoscale, ${ }^{[9,10,15]}$ and as a demonstrative example, the periodic arrangement of gold nanoparticles is shown in Fig. 5. ${ }^{[16]}$ The network consists of a two tile-system (brown and blue in Fig. 5a and 5b), from which the blue tiles are exposing an additional 15 base-pair long single strand DNA (red in Fig. 5a and 5b). Upon addition of gold nanoparticles (5 $\mathrm{nm}$ in diameter) exposing the complementary single-stranded DNA, exclusively the blue tiles should be decorated with a gold nanoparticle. To the surprise of the authors, a periodic square lattice arrangement of the nanoparticles with a lateral spacing of $38 \mathrm{~nm}$ was observed, showing that only every second potential immobilization knot is involved in the immobilization of the particles. Both the net-type character of the DNA-tile architecture (Fig. 5c) and the spacing of the gold nanoparticles (Fig. 5d) are beautifully visualized by their AFM graphs.

In summary, the impressive level of control already encoded in the parent building blocks made DNA-based architectures a perfect tool to control the spatial arrangement at the nanoscale. The obtained fabrics are mainly used to laterally organize the objects of interest and have many features in common with our everyday macroscopic fabrics. However, with their mechanically interlinked subunits that self-assemble into periodic 2D patterns by supramolecular interactions, they rather resemble a fishing net with fixed knots at the junction of two yarns than an interwoven fabric with simply mechanically interwoven yarns.

\subsection{Coordination Polymers Forming 2D Fabrics}

Another class of supramolecular structures resembling interwoven fabrics are coordination polymers. The combination of interlinking ligands exposing two terminal binding sites with metal ions suitable to connect two ligands can yield in 1D chains structure programmed in the sequences of the DNA strands. Adapted from ref. [13] with permission from Springer Nature. 

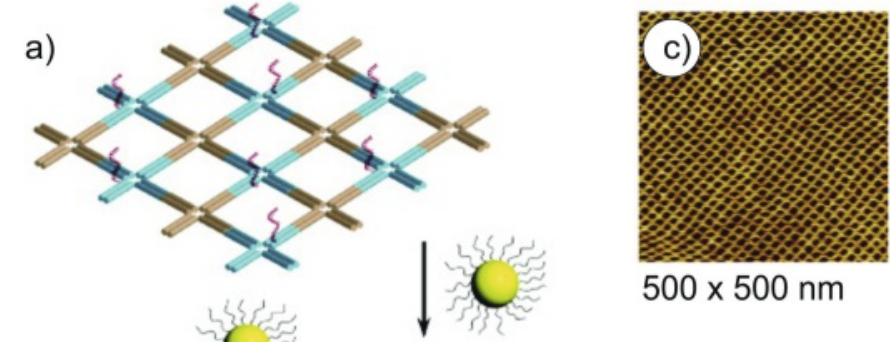

$500 \times 500 \mathrm{~nm}$
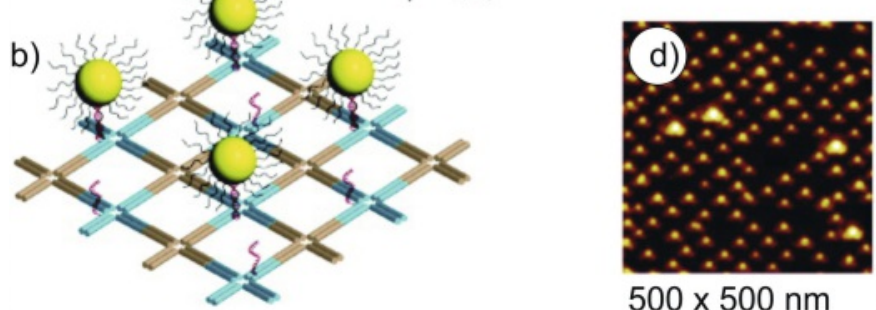

$500 \times 500 \mathrm{~nm}$

Fig. 5. a) Sketch of the DNA network obtained by a two tiles (brown and blue) from which only the blue tiles are exposing a 15-base pairs long single strand DNA (red). b) Immobilization of $5 \mathrm{~nm}$ large gold nanoparticles exposing complementary DNA strands. c) AFM picture of the DNA lattice before the addition of gold nanoparticles. d) AFM height picture of the gold nanoparticles assembled on the 2D DNA lattice. Reprinted with permission from ref. [16]. Copyright 2019 American Chemical Society.

of so-called coordination polymers.[17] While ligands and metal ions are dissolved, the macromolecular coordination polymers in many cases lack solubility and often conditions were chosen to allow their ordered arrangement by crystallization. The obtained single crystals are tightly packed and there are several reported cases where the 1D coordination polymer chains are interwoven in $2 \mathrm{D}$ sheets. In the following, the analogy of the observed arrangement with 2D fabrics is discussed with a few examples. However, due to the solid-state nature of the objects, there are hardly any particular features arising from the interwoven arrangement and the resemblance to $2 \mathrm{D}$ fabrics is restricted to the structural similarity.

An early illustrative example is the formation of an interwoven textile-type structure based on a gold-gold interactions. ${ }^{[18]}$ The monomer consisting of two 1,6-bis(diphenylphosphino)hexane interlinked gold iodine centres $\left((\mathrm{AuI})_{2}(\mu\right.$-dpph)) crystallizes from a dichloromethane-methanol mixture in long chains with gold-gold contacts (Fig. 6a). In the solid-state structure of the compound, these 1D-chains are arranged in 2D-sheets of interwoven chains. The biaxially woven textile type arrangement is sketched in Fig. 6b, while Fig. 6c displays a side-view of the $\mathrm{X}$-ray structure displaying the arrangement in discrete $2 \mathrm{D}$ sheets.

Other examples of biaxial interwoven chains of coordination polymers are the solid-state structure obtained from 1-(isocyanidomethyl)-1 $H$-benzotriazole and $\mathrm{Ag}(\mathrm{I})$ ions, ${ }^{[19]}$ or the 'warp-and-woof' sheets consisting of 4,4'-bisazobipyridine interlinked $\mathrm{Cu}$ ions exposing 2,2'-bipyridine corner units. [20]

In terms of atom diversity an even simpler compound is the solid-state structure obtained by the reaction of gold with sodium polysulfide liquids. [21] $1 \mathrm{D}$ infinite chains consisting of sulphur interlinked gold atoms $\left((\mathrm{AuS})_{\mathrm{n}}{ }^{\mathrm{n}}\right)$ ) are formed, which arrange in parallel sheets separated by sodium cations. The $(\mathrm{AuS})^{\mathrm{n}}{ }^{\mathrm{n}}$ - chains coil forming an interwoven layer structure resembling a "chickenwire'. The arrangement enables to establish interchain $\mathrm{Au}-\mathrm{Au}$ contacts favouring the formation of these 'chicken-wire' fabrics. Fig. 7 displays the anionic 2D interwoven sheet with the X-ray structure at the left side and differently coloured 'chicken-wire'type interwoven $1 \mathrm{D}$ strains at the right side.

Another fascinating example of mechanically interlocked 2D sheet has been observed in the solid-state structure of the coordination polymer $\operatorname{Ag}_{2}$ (1,4-bis(imidazol-1-ylmethyl)

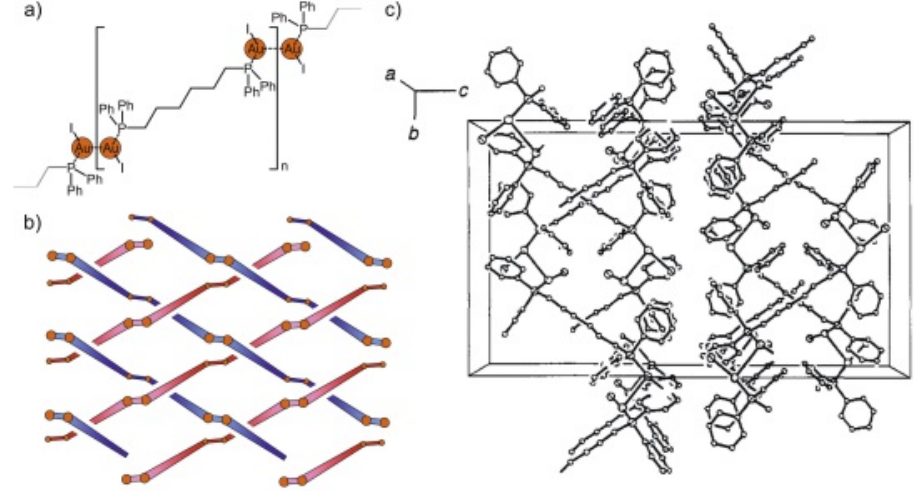

Fig. 6. a) The coordination polymer chain formed by Au-Au bonds of $(A u l)_{2}(\mu-d p p h)$. The Au-atoms are displayed as orange circles. b) Sketch of the biaxial interwoven structure. The perpendicular strands are displayed in red and blue respectively. c) Side-view of the solidstate structure displaying the 2D sheets consisting of the interwoven coordination polymer. Adapted from ref. [18] with permission from the Royal Society of Chemistry.

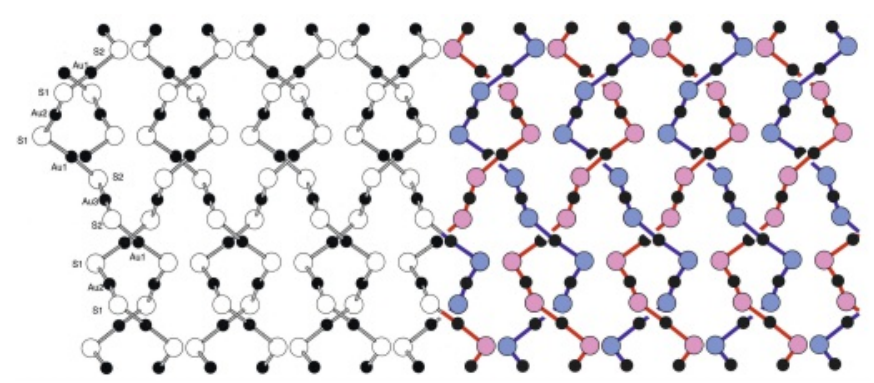

Fig. 7. 'Chicken-wire'-type interwoven network formed by (AuS) ${ }_{n}{ }^{n-}$ chains. The left side display the solid-state structure obtained by X-ray diffraction and the differently coloured 1D chains on the right side improve the visibility of the 'chicken-wire'-type interwoven network. Adapted with permission from ref. [21]. Copyright 2019 American Chemical Society.

benzene $)_{3}\left(\mathrm{NO}_{3}\right)_{2} \cdot{ }^{[22]}$ The compound crystallizes in infinite chains consisting of threefold-coordinated $\mathrm{Ag}(\mathrm{I})$ ions. As displayed in Fig. 8a), the threefold coordination sphere at the Ag ions is obtained by alternating single ligands with pairs of ligands forming macrocycles. The void opened by the macrocycles is filled by monomeric ligand sequences of perpendicular chains such that a 2D network of 'rotaxanated' (mechanically interlocked) chains is formed. In this fascinating arrangement (Fig. 8b) each chain of a sheet mechanically interacts with every perpendicular chain exactly once with alternating role as axes or macrocycle. To visualize the arrangement in the 2D sheet, the solid-state structure is displayed in Fig. 8b. As sketched in Fig. 8c and in analogy to biaxial woven fabrics, the sheets consist of mechanically fixed perpendicular chains but differ in the nature of the mechanical link.

Another type of interwoven 2D networks has been observed for the coordination polymer displayed in Fig. 9a). ${ }^{[23]}$ The bifunctional and chiral ligand coordinates with metal ions ( $\mathrm{Zn}$ (II) or $\mathrm{Cd}(\mathrm{II})$ ) forming wavy grids with the coordinated ions as knot surrounded by four ligands (Fig. 9b). A pair of grids forms an interwoven 2D sheet, described by the authors as parallel double helices (Fig. $9 \mathrm{c})$ interconnected by the metal centres. The interwoven sheet formation by interlinked double helices is conceptually visualized in Fig. 9d.

Interwoven grids were also observed for a coordination polymer consisting of $\mathrm{Zn}$ (II) ions as knots, which were interlinked by muconate $((E, E)$-2,4-hexadienedioate) and 4,4'-bisazobipyridine ligands. ${ }^{[24]}$ As displayed in Fig. $10 a$, the combination of both ligands formed grids consisting of parallel chains of muconate interlinked 

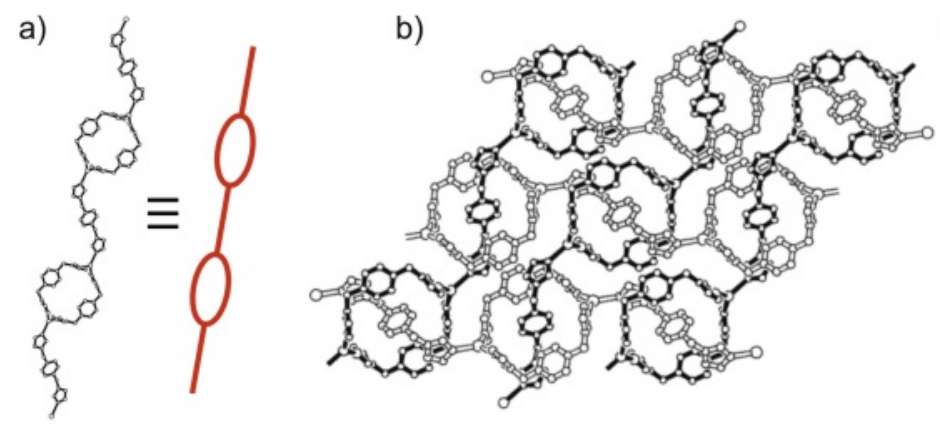

a)

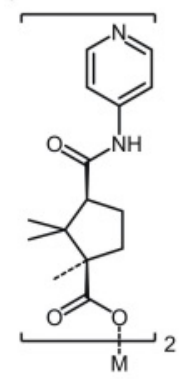

$\mathrm{M}=\mathrm{Zn}(\mathrm{II})$ or $\mathrm{Cd}(\mathrm{II})$ b)

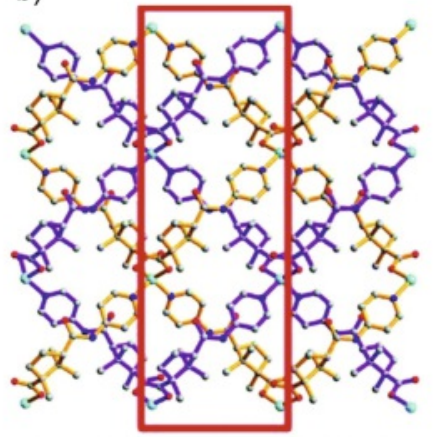

c)

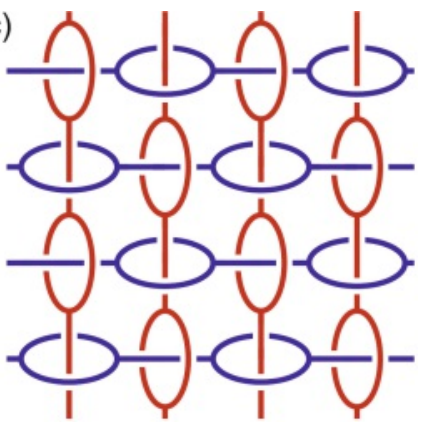

c)

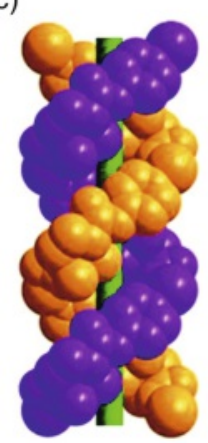

d)

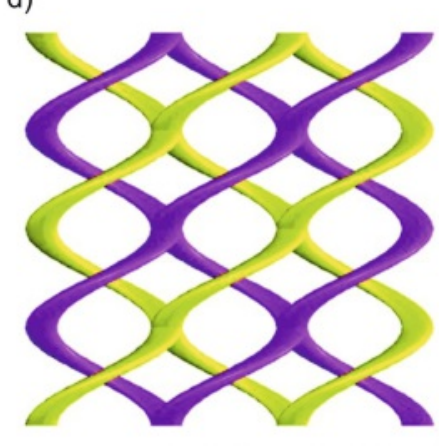

Fig. 8. Infinite 2D polyrotaxane network. a) X-ray structure and sketch of the 1D polymer chain. b) Solid-state structure of the polyrotaxane framework. c) Sketch of the mechanically interlocked 1D chains forming a 2D sheet. Adapted with permission from ref. [22]. Copyright 2019 American Chemical Society. zinc centres, which were interconnected by 4,4 '-bisazobipyridine ligands. To avoid empty space in the solid-state structure, two grids form a 2D sheet of interwoven grids (Fig. 10b).

Both last examples differ from fabrics as their 2D spread is provided by the grid-type coordination polymer and is not a result of the pairwise interwoven arrangement.

In summary, there are numerous coordination polymers with spatial arrangements in the solid-state resembling aspects of interwoven fabrics. However, the solid-state nature of the materials handicaps the exploitation of their mechanical, fabriclike features.

There are many more reported examples of interwoven networks ${ }^{[25,26]}$ and the examples discussed here are more the representatives of a particular type of $2 \mathrm{D}$ fabric-type arrangement than a comprehensive collection. Also most examples report 3D networks, with 3D coordination polymers or metal organic frameworks (MOF) an emerging and fast growing research field of its own.[27-29] While there are numerous examples of interpenetrated 3D MOF structures ${ }^{[30]}$ and their discussion is clearly beyond the scope of this article, MOFs are promising a)

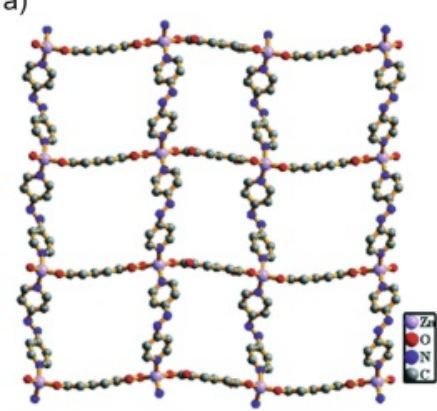

b)

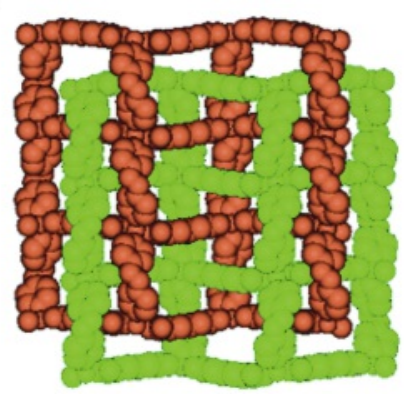

Fig. 10. a) Solid-state structure of the $2 \mathrm{D}$ coordination polymer with $\mathrm{Zn}(\mathrm{II})$ muconate chains arranged horizontally and the interconnecting 4,4'-bisazobipyridines vertically. b) Closely-packed interwoven arrangement of two 2D grids (green and brown). Adapted from ref. [24] with permission from the Royal Society of Chemistry. scaffolds with the potential to gain spatial control at the molecular level.

\subsection{Fabrics Based on Tailor-made Organic Structures}

Also the DNA-based lattices discussed in section 3.1 could be classified as being obtained from tailor-made organic structures. Here we would like to distinguish between the biomoleculeinspired strategies presented in section 3.1 profiting from the tuneable intermolecular attraction of DNA base pairing and concepts geared towards interlinking of purpose-designed and synthesized small molecules.

As presented in the article so far, there are numerous sophisticated strategies resulting in 2D interwoven frameworks. A major challenge for concepts aiming towards interwoven linear molecules is the high level of static and dynamic structural control required. A quickly growing number of 3D covalent organic frameworks $(\mathrm{COF})^{[31-33]}$ and $2 \mathrm{D}$ polymers ${ }^{[34,35]}$ documents that the controlled growth is not limited to 1D polymer chains, strategies resulting in interwoven networks are still rare.

A first example profits from metal complexes to provide spatial control and predictable crossing geometries, ${ }^{[36]}$ a strategy that was already applied successfully above for the assembly of Solomon knots. ${ }^{[6]}$ The $\mathrm{Cu}$ (II) bis-phenantroline complex displayed in Fig. 11a exposes four aldehydes in a 'tetrahedral' geometry, which form a crystalline 3D COF network upon imine formation with 4,4'-diaminobiphenyl. The COF consists of linear polymers of imine interlinked alternating phenantroline and biphenyl subunits and due to the $\mathrm{Cu}$ (II) phenantroline complexes, crossing polymer chains are mechanically interlinked upon removal of the coordinating ions (Fig. 11b). The microcrystalline samples obtained were analyzed by powder X-ray diffraction (PXRD) techniques proving the periodic arrangement of the metal centers, but with limited sensitivity with respect to the perfection of the 3D interwoven organic network (Fig. 11b and 11c). The interwoven 3D network structure is likely to persist even with defects along the polymer chains. However, the mechanical properties of the minute samples obtained support their interwoven character as a tenfold increase in their elasticity was recorded compared to 
the micro crystallites of the initial COF comprising the copper ions. Following the same strategy but using a larger counter ion for the $\mathrm{Cu}$ (II) phenantroline starting material, the ability of the resulting 3D interwoven network to accept larger guests was even improved. ${ }^{[37]}$ Using cross-shaped tetraamine structures to interlink the copper phenantroline complexes resulted, after demetalation, in 3D frameworks consisting of mechanically interlinked 1D chains of macrocycles. ${ }^{[38]}$

These pioneering examples of mechanically interlinked polymer chains have the interwoven character in common with fabrics but differ in their expansion. While fabrics are twodimensional arrangements, these COFs are bulk materials spreading out in three dimensions.

Molecular 2D fabrics require the arrangement of linear polymer chains in an interwoven fashion. In analogy to the loom that properly arranges the yarns resulting in a 2D textile, a tool organizing the linear polymer on a molecular level is required. As a potential technique that could provide the ordering power are the layer-by-layer grown metal organic frameworks, so called SURMOFs developed by Christof Wöll.[39,40] The layerby-layer architecture enables the separation of particular layers and the fixation of the organic linker molecules as struts in the separated MOF plane can be used as preorganization for the fabric formation. As sketched in Fig. 12, a SURMOF was grown on a gold substrate consisting of layers of copper ion-interlinked 4,4"-para-terphenyl dicarbocylic acids forming squares. ${ }^{[41]}$ The special MOF layers that shall form molecular fabrics were assembled with a tailor-made terphenyl dicarboxylic acid. This strut exposes on both sides perpendicular chains with a terminal acetylene (green in Fig. 12). After its fixation in the MOF plane, the MOF was exposed to reaction conditions enabling the crosslinking of the struts facing each other in the MOF squares by Glaser-type oxidative acetylene coupling forming linear polymers of interconnected MOF struts. The flexibility of the structures forming the polymer enabled the crosslinking of both pairs of struts to form a square of the MOF plane. The dimension of the polymer-forming structure was slightly too large for the MOF square, such that the connection was either formed above or below the MOF plane. As the polymer is mounted on the strut as rotation axis, a polymer connection in one square above the MOF plane forced the connection in the neighbouring square below the plane and vice versa. A connection of a vis-à-vis pair of

a)

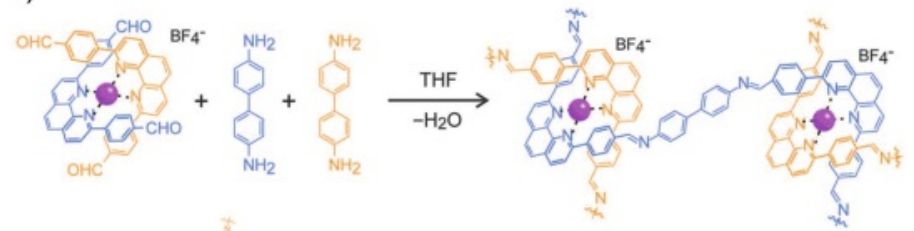

b)

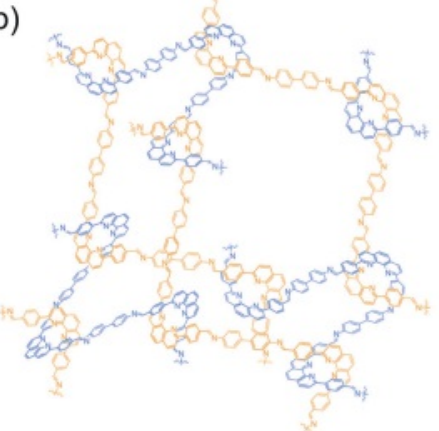

c)

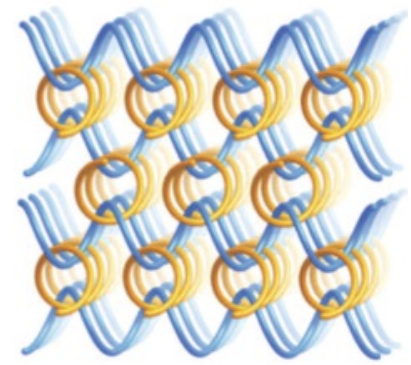

Fig. 11. 3D network consisting of interwoven linear polymers. a) Condensation reaction forming the COF consisting of interlinked copper phenantroline complexes. b) Molecular drawing of the interwoven polymer chains. c) Sketch of the 3D material consisting of interwoven 1D-polymer chains. Adapted from ref. [36] with permission from AAAS. struts above the MOF plane further forced the second connection in the same MOF square below the plane. Considering these geometrical restrictions, a complete polymerization within the MOF plane should result in biaxial interwoven linear polymers. Upon disintegration of the MOF framework by demetalation, the molecular textile was released.

While the chemical transformation was spectroscopically observed in the crystalline SURMOF, flakes of molecular textiles down to three molecular layers thick were obtained and characterized by scanning electron microscopy (SEM) and AFM. The interwoven nature of these flakes was demonstrated by disassembling the $2 \mathrm{D}$ fabrics into individual polymer strands.

The major challenges to improve the dimension of the accessible fabrics are not only the quality of the single crystalline MOF plane, but also the control over the extent of linear polymer formation. To address the latter issue we are currently exploring the potential of reversible coupling chemistry.

Another elegant approach to interwoven organic fabrics is based on supramolecular interactions of a rigid oligoproline rod exposing two perylenemonoimides at a distance of about $18 \AA$ to the same side (Fig. 13a). ${ }^{[42]}$ The $\pi$-stacking between these perylenemonoimine subunits results in the formation of long linear threads with a regular periodicity of up and down oriented voids (Fig. 13b). To avoid empty voids the compound self-assembles into a triaxial interwoven network with the three crossing threads filling each other's voids (Fig. 13c). The resulting superstructure is a Kagome lattice of several micrometres in dimension, which can clearly be recognized by tunnelling electron microscopy (TEM, Fig. 13d). The system beautifully profits from the balanced interactions. While the threads formed by supramolecular forces probably have limited mechanical tensile strength, they enable the large scale self-assembly to regular interwoven $2 \mathrm{D}$ patterns.

\section{Conclusion and Outlook}

The collection of examples illustrate how interwoven fabrics and their properties inspired chemists in their search for new functions emerging from the molecular structure. While discrete (supra)molecular structures can be assembled to prove the validity of the concept, the fabrication of $2 \mathrm{D}$ molecular textiles of considerable dimensions remains challenging. In the case of the DNA-based self-assembled structures the framework itself becomes the tool enabling spatial control over nanoscale entities. However, the networks in most cases lack the lateral flexibility of textiles emerging from the sliding of the yarns at the intersection.

a)
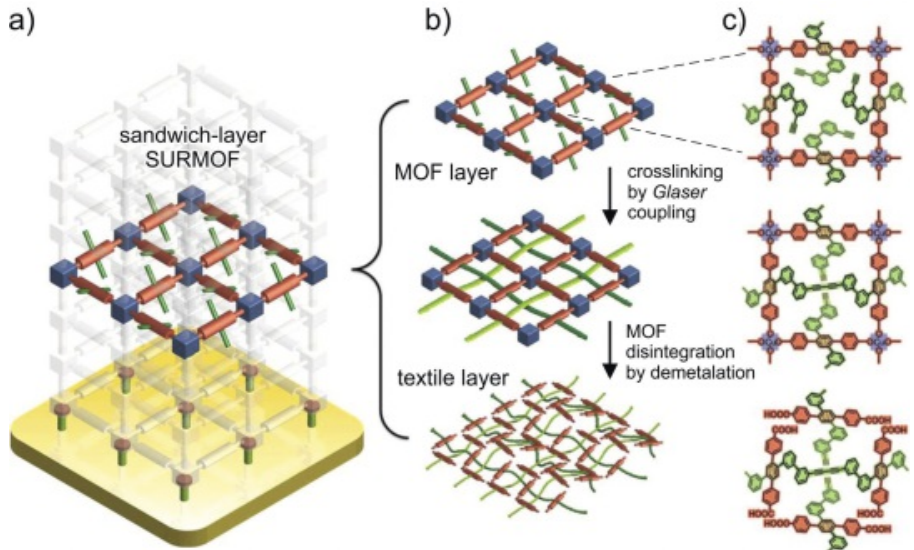

Fig. 12. Formation of a molecular textile in a layer of a SURMOF. a) Sandwich layer of the polymer precursor struts mounted in a SURMOF. b) Top: the sandwich layer fixed in the SURMOF. Middle: Linear polymers (green strands) formed upon crosslinking of the struts. Bottom: Release of the molecular textile upon disintegration of the SURMOF. c) Chemical transformations in a unit cell of the SURMOF plane. Adapted from ref. [41]. 

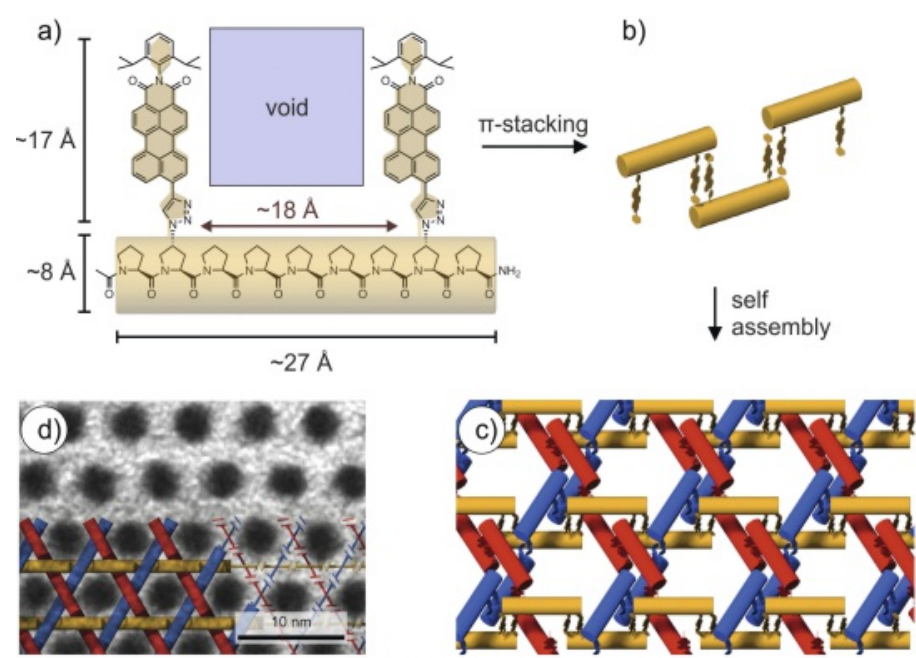

Fig. 13. The triaxial supramolecular Kagome lattice. a) Molecular design and dimensions of the oligoproline exposing two perylenemonoimides defining an empty void in between both chromophores. b) Formation of the threads by intermolecular $\pi$-stacking. c) Self-assembly into the triaxial weave forming the Kagome-lattice. d) TEM micrograph of the Kagome-lattice with the sketch of the molecular structure overlaid. Adapted from ref. [42] with permission from Springer Nature.

The examples of interwoven coordination polymers usually share exclusively the structural analogy to textiles and fabrics but barely profit from the interwoven spatial arrangement of the 1D-polymer chains due to the solid-state nature of the material. The rather recent approaches to assemble molecular weaves by suitably pre-organized small organic molecules are promising proofs of concept with considerable development potential.

In our own approach using a SURMOF to organize the polymer precursors, the dimensions of the obtained molecular textiles are limited. We are currently exploring the suitability of the air/water interface for the formation of molecular textiles with suitable supramolecular building blocks. While preliminary experiments are promising, the major challenge is the monomolecular nature of the sheet resulting in very moderate spectroscopic signals making its analysis and characterization demanding.

Strategies enabling the controlled interweaving of linear polymers remain an open challenge and a worthwhile aim, as the obtained molecular fabrics are likely to have interesting physical properties emerging form the mechanical nature of the cohesion of their subunits.

\section{Acknowledgements}

Financial support by the Swiss National Science Foundation (SNF grant number 200020-178808) and the 111 project (90002-18011002) is gratefully acknowledged.
[1] P. D. Dubrovski, 'Woven Fabric Engineering', IntechOpen, 2010.

[2] M. Akbari, A. Tamayol, S. Bagherifard, L. Serex, P. Mostafalu, N. Faramarzi, M. H. Mohammadi, A. Khademhosseini, Adv. Healthc. Mater. 2016, 5, 751.

[3] V. B. C. Tan, X. S. Zeng, V. P. W. Shim, Int. J. Impact Eng. 2008, 35, 1303.

[4] S. Adanur, 'Handbook of Weaving', CRC Press, n.d.

[5] K. Gandhi, 'Woven Textiles: Principles, Technologies and Applications', Elsevier, 2012.

[6] J. F. Nierengarten, C. O. Dietrich-Buchecker, J. P. Sauvage, J. Am. Chem. Soc. 1994, 116, 375 .

[7] C. D. Pentecost, K. S. Chichak, A. J. Peters, G. W. V. Cave, S. J. Cantrill, J. F. Stoddart, Angew. Chem. Int. Ed. 2007, 46, 218.

[8] T. Ciengshin, R. Sha, N. C. Seeman, Angew. Chem. 2011, 123, 4511.

[9] M. R. Jones, N. C. Seeman, C. A. Mirkin, Science 2015, 347, 1260901.

[10] F. Hong, F. Zhang, Y. Liu, H. Yan, Chem. Rev. 2017, 117, 12584.

[11] J. Chen, N. C. Seeman, Nature 1991, 350, 631

[12] E. Winfree, F. Liu, L. A. Wenzler, N. C. Seeman, Nature 1998, 394, 539.

[13] P. W. K. Rothemund, Nature 2006, 440, 297.

[14] J. Malo, J. C. Mitchell, C. Vénien-Bryan, J. R. Harris, H. Wille, D. J. Sherratt, A. J. Turberfield, Angew. Chem. Int. Ed. 2005, 44, 3057.

[15] C. Lin, Y. Liu, S. Rinker, H. Yan, ChemPhysChem 2006, 7, 1641.

[16] J. Zhang, Y. Liu, Y. Ke, H. Yan, Nano Lett. 2006, 6, 248.

[17] W. L. Leong, J. J. Vittal, Chem. Rev. 2011, 111, 688.

[18] P. M. Van Calcar, M. M. Olmstead, A. L. Balch, J. Chem. Soc. Chem. Commun 1995, 1773 .

[19] I. Ino, J. C. Zhong, M. Munakata, T. Kuroda-Sowa, M. Maekawa, Y. Suenaga, Y. Kitamori, Inorg. Chem. 2000, 39, 4273.

[20] L. Carlucci, G. Ciani, A. Gramaccioli, D. M. Proserpio, S. Rizzato, CrystEngComm 2000, 2, 154.

[21] E. A. Axtell, J.-H. Liao, M. G. Kanatzidis, Inorg. Chem. 1998, 37, 5583.

[22] B. F. Hoskins, R. Robson, D. A. Slizys, J. Am. Chem. Soc. 1997, 119, 2952.

[23] Q. Huang, J. Yu, J. Gao, X. Rao, X. Yang, Y. Cui, C. Wu, Z. Zhang, S. Xiang, B. Chen, G. Qian, Cryst. Growth Des. 2010, 10, 5291.

[24] C. M. Nagaraja, B. Ugale, A. Chanthapally, CrystEngComm 2014, 16, 4805

[25] S. R. Batten, R. Robson, Angew. Chem. Int. Ed. 1998, 37, 1460.

[26] Y. Liu, M. O'Keeffe, M. M. J. Treacy, O. M. Yaghi, Chem. Soc. Rev. 2018, $47,4642$.

[27] H. Furukawa, K. E. Cordova, M. O’Keeffe, O. M. Yaghi, Science 2013, 341, 1230444.

[28] H.-C. Zhou, J. R. Long, O. M. Yaghi, Chem. Rev. 2012, 112, 673.

[29] S. Yuan, L. Feng, K. Wang, J. Pang, M. Bosch, C. Lollar, Y. Sun, J. Qin, X. Yang, P. Zhang, Q. Wang, L. Zou, Y. Zhang, L. Zhang, Y. Fang, J. Li, H. C. Zhou, Adv. Mater. 2018, 30, 1704303.

[30] Y.-N. Gong, D.-C. Zhong, T.-B. Lu, CrystEngComm 2016, 18, 2596.

[31] S.-Y. Ding, W. Wang, Chem. Soc. Rev. 2012, 42, 548.

[32] P. J. Waller, F. Gándara, O. M. Yaghi, Acc. Chem. Res. 2015, 48, 3053.

[33] M. S. Lohse, T. Bein, Adv. Funct. Mater. 2018, 28, 1705553.

[34] W. Wang, A. D. Schlüter, Macromol. Rapid Commun. 2019, 40, 1800719.

[35] J. Sakamoto, J. van Heijst, O. Lukin, A. D. Schlüter, Angew. Chem. Int. Ed. 2009, 48, 1030.

[36] Y. Liu, Y. Ma, Y. Zhao, X. Sun, F. Gandara, H. Furukawa, Z. Liu, H. Zhu, C. Zhu, K. Suenaga, P. Oleynikov, A. S. Alshammari, X. Zhang, O. Terasaki, O. M. Yaghi, Science 2016, 351, 365.

[37] Y. Liu, Y. Ma, J. Yang, C. S. Diercks, N. Tamura, F. Jin, O. M. Yaghi, J. Am. Chem. Soc. 2018, 140, 16015.

[38] Y. Liu, C. S. Diercks, Y. Ma, H. Lyu, C. Zhu, S. A. Alshmimri, S. Alshihri, O. M. Yaghi, J. Am. Chem. Soc. 2019, 141, 677.

[39] O. Shekhah, H. Wang, S. Kowarik, F. Schreiber, M. Paulus, M. Tolan, C. Sternemann, F. Evers, D. Zacher, R. A. Fischer, C. Wöll, J. Am. Chem. Soc. 2007, 129, 15118 .

[40] J.-L. Zhuang, A. Terfort, C. Wöll, Coord. Chem. Rev. 2016, 307, 391.

[41] Z. Wang, A. Błaszczyk, O. Fuhr, S. Heissler, C. Wöll, M. Mayor, Nat. Commun. 2017, 8, 14442 .

[42] U. Lewandowska, W. Zajaczkowski, S. Corra, J. Tanabe, R. Borrmann, E. M. Benetti, S. Stappert, K. Watanabe, N. A. K. Ochs, R. Schaeublin, C. Li, E. Yashmina, W. Pisula, K. Müllen, H. Wennemers, Nat. Chem. 2017, 9, 1068. 